\title{
Concurrent Hydatidosis of the Liver, Spleen and Breast: A Case Report
}

\author{
Arash Arianpoor ${ }^{1}$, Mohammad Reza Motie ${ }^{1,2,{ }^{*}}$, Mahdi Yousefzadeh Telefoni ${ }^{1}$ and Elahe Amini ${ }^{1}$ \\ ${ }^{1}$ Surgical Oncology Research Center, Mashhad University of Medical Sciences, Mashhad, Iran \\ ${ }^{2}$ Department of Surgery, Imam Reza Hospital, Mashhad University of Medical Sciences, Mashhad, Iran \\ "Corresponding author: Department of Surgery, Imam Reza Hospital, Ahmadabad St., Mashhad, Iran. Tel: +98-9151101871, Email: motiem@mums.ac.ir
}

Received 2018 July 14; Revised 2018 July 14; Accepted 2018 August 12.

\begin{abstract}
Introduction: Hydatid disease is an infection caused by the larval form of Echinococous granulosus and is endemic in animal husbandry communities. Hydatid disease of the breast is rare and occurs in less than $1 \%$ of the cases. In this paper, we presented a known case of hepatic hydatidosis readmitted due to hydatid cyst of the breast.

Case Presentation: A 38-year-old woman, a known case of neglected hepatic hydatidosis since eight years ago, presented to our hospital with new onset of abdominal pain. Computed tomography (CT) scan revealed signs of hydatid cysts in the liver, spleen, and both breasts. Laparotomy with complete resection of the hydatid cysts in the right lobe of the liver was performed along with cholecystectomy due to the closeness of the cysts to the gallbladder and severe adhesions. The patient also underwent splenectomy and cystectomy of the breasts. Histopathologic report of the resected cysts from the breasts, liver, and spleen confirmed the diagnosis of hydatidosis. The patient was discharged with albendazole $(10 \mathrm{mg} / \mathrm{kg}$ daily for three weeks) and was advised to come back to the clinic for follow-up evaluations.

Conclusions: Although breast hydatidosis is rare, it should be considered as a differential diagnosis of breast lumps presenting in endemic areas and among immigrants from endemic areas.
\end{abstract}

Keywords: Hydatidosis, Hepatic Echinococcosis, Breast Cyst, Echinococcus granulosus

\section{Introduction}

Hydatid disease is an infection caused by Echinococous granulosus. Despite the fact that humans are considered as accidental intermediate hosts of this parasite, cystic echinococcosis (CE) could be fatal (1). This zoonotic disease, associated with poverty and poor hygiene, is especially endemic in animal husbandry communities with low-income (2). Age, dog ownership, residence area, and educational level are believed be significantly associated with echinococcosis (3). The rate of hydatid disease is high in different parts of Asia including the Middle East, and especially in Iran, and is even hyper-endemic in some areas (4-8).

The liver is the most common site to be affected by this disease (70\%), followed by the lungs (20\%) (6). Hydatid disease of breast is rare and occurs in less than $1 \%$ of the cases $(5,7,9,10)$. In this paper, we presented a known case of hepatic hydatidosis readmitted due to hydatid cyst of the breast.

\section{Case Presentation}

A 38-year-old woman, who was a known case of hydatid cyst of the liver since eight years ago, presented with new onset of abdominal pain in the right upper quadrant (RUQ) and the left upper quadrant (LUQ). The patient was a candidate for cystectomy, but refused to undergo liver resection. She also neglected her medical treatment. She presented to our hospital with the aforementioned abdominal pain for the first time after her initial diagnosis.

In the process of her evaluation, computed tomography (CT) scan was performed, which was not only suggestive of hydatid cysts in the right lobe of the liver and spleen (Figure 1), but also accidentally revealed two suspicious masses in the left breast and another in the right one (Figure 2). The size of the breast cysts was estimated to be 35 $\times 35$ and $25 \times 31 \mathrm{~mm}$ in the upper lateral and lower lateral quadrants of the left breast and $31 \times 31 \mathrm{~mm}$ in the upper lateral quadrant of the right breast. The results of blood analysis and chest radiograph were normal.

The patient underwent surgery and the breast cysts were resected (Figure 3). Laparotomy followed by total 


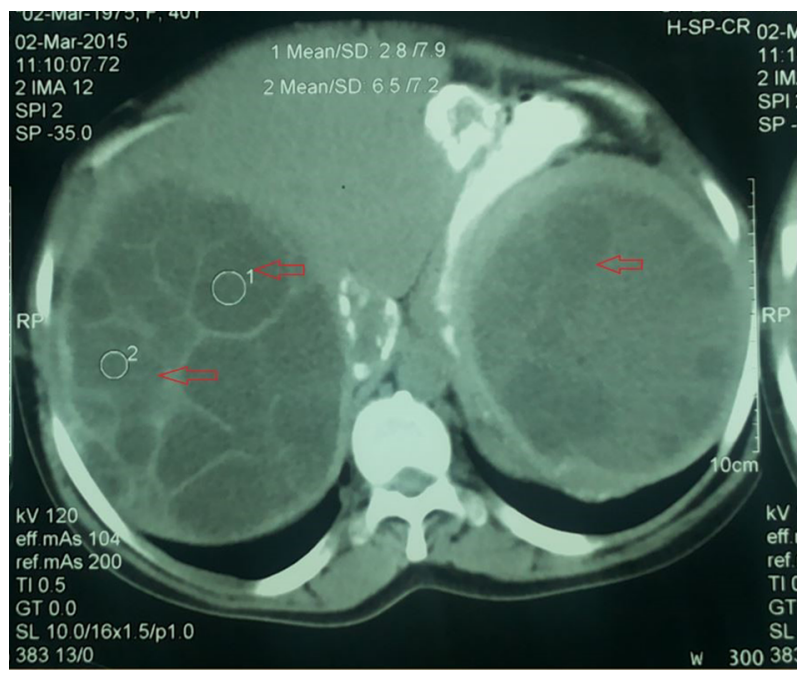

Figure 1. Liver and spleen cysts

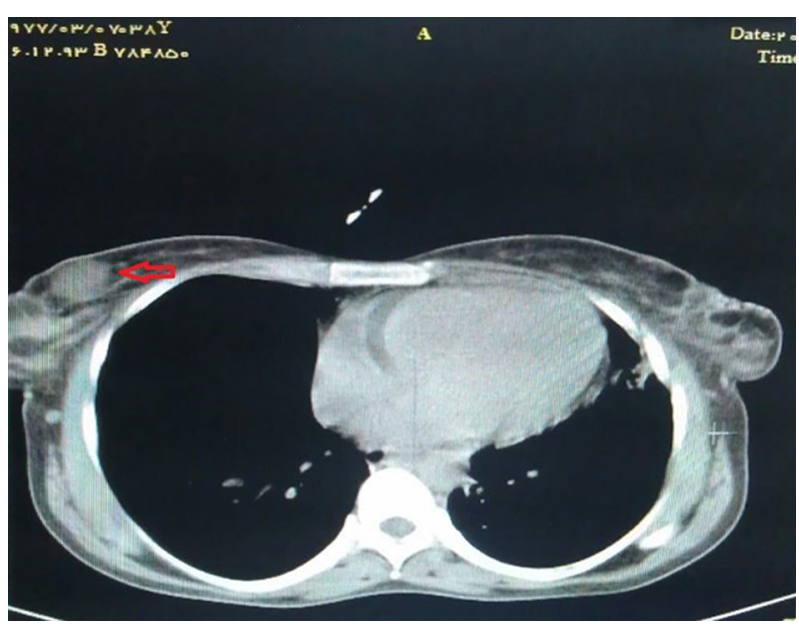

Figure 2. Breast cysts

splenectomy and complete resection of the liver cysts was also performed. In addition, cholecystectomy was carried out due to the closeness of the cysts to the gallbladder and severe adhesions to the adjacent tissue.

Histopathologic studies of the resected cysts and cyst materials confirmed the diagnosis of hydatid disease. Finally, the patient was discharged with albendazole (10 $\mathrm{mg} / \mathrm{kg}$ daily for three weeks) and was advised to come back for further follow-up.

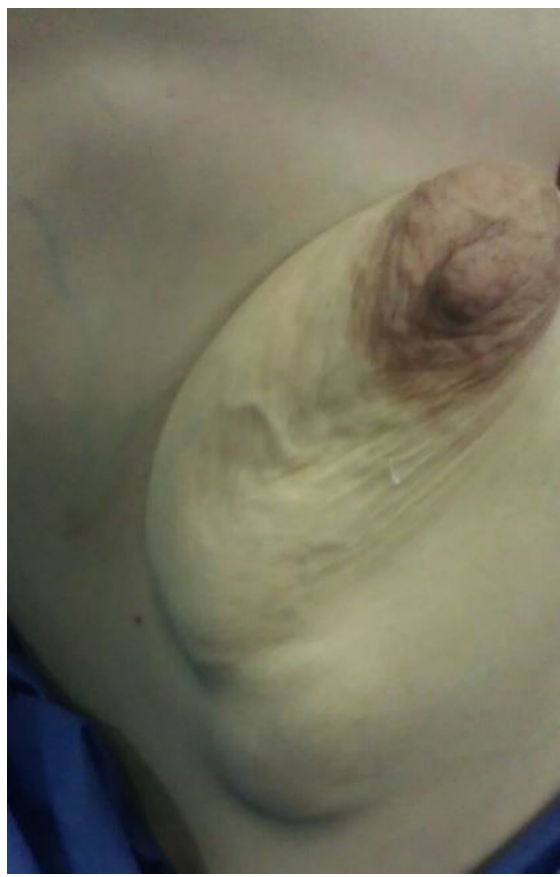

Figure 3. The breast after cystectomy

\section{Discussion}

Cystic echinococcosis is a parasitic infection caused by several Echinococcus species (11). The adult tapeworm lives in the small intestine of canines (the definitive host). Its reproductive segments (proglottids) are released in the feces. The intermediate hosts (e.g., sheep, goat, and cattle) are then infected by ingesting contaminated plants. After entering the ruminant's gut, the egg hatches and releases an oncosphere, which penetrates the intestinal wall, migrates to different organs (especially the liver and lungs) and there develops into a cyst. The cycle is completed when the definitive host ingests the cyst-containing organs of the intermediate host $(12,13)$.

Humans are considered as an incidental host, acquiring the infection mainly by consumption of contaminated vegetables and water. Similar to intermediate hosts, ingested larvae penetrate the systemic or lymphatic circulation from the intestinal mucosa and finally reside in different organs, mostly the liver and lungs $(6,13)$. Rarely, the larvae may also infest the muscles, kidneys, spleen, bones, and breasts $(5,7,9,10,14)$.

Hydatid disease of the breast is rare and accounts for less than $1 \%$ of all cases $(5-7,10)$. Cystic echinococcosis usually involves the breast as the secondary site following hematologic or lymphatic dissemination. However, 
rarely breasts can be the primary site of hydatidosis $(15,16)$. The typical presentation of breast hydatidosis is painless slow growing breast lump without regional lymph node involvement. It generally affects women between 30 - 50 years of age. It might mimic fibroadenoma, phyllodes tumors, chronic abscess, or even carcinoma. Thus, hydatid cyst of the breast should be included in the differential diagnosis of breast lumps, especially in endemic areas (4, 9 ). The disease can be diagnosed by radiologic or serologic means, neither of which is definitive. The diagnosis of hydatid disease is based on the findings of enzyme-linked immunosorbent assay (ELISA) for echinococcal antigens and results are positive in approximately $85 \%$ of infected patients $(9,12,17)$. In patients with intact cysts or without any scolices or any viable parasites, ELISA results may be negative. Serologic tests such as intradermal and indirect hemagglutination tests may be used to confirm the diagnosis $(4,5)$.

Mammography may show the characteristic ring shape structures inside the mass, which strongly suggests hydatid cysts of the breast $(4,18)$. Ultrasound and magnetic resonance imaging are helpful diagnostic tools. Although fine needle aspiration biopsy in suspected lesions may confirm the diagnosis by observing the hooklets of the parasite, it should be avoided due to the high risk of anaphylactic reaction (5).

\subsection{Conclusion}

Although hydatid cyst of the breast is rare, it should be considered as a differential diagnosis of breast lumps, especially in endemic areas and among immigrants from endemic areas. In the breast, hydatidosis may mimic a simple cyst, fibroadenoma, chronic abscess, phyllodes tumor, or even carcinoma. Both serologic (such as ELISA) and radiologic studies (such as mammography, ultrasonography, and CT scan) can be helpful to establish the diagnosis of hydatid disease. Finally, surgical removal without spillage is the best method of the treatment of hydatid lesions.

\section{References}

1. Nikmanesh B, Mirhendi H, Mahmoudi S, Rokni MB. Multilocus sequence analysis of Echinococcus granulosus strains isolated from humans and animals in Iran. Exp Parasitol. 2017;183:50-5. doi: 10.1016/j.exppara.2017.10.002. [PubMed: 29024693].
2. Cucher MA, Macchiaroli N, Baldi G, Camicia F, Prada L, Maldonado L, et al. Cystic echinococcosis in South America: Systematic review of species and genotypes of Echinococcus granulosus sensu lato in humans and natural domestic hosts. Trop Med Int Health. 2016;21(2):16675. doi: 10.1111/tmi.12647. [PubMed: 26610060].

3. Galeh TM, Spotin A, Mahami-Oskouei M, Carmena D, Rahimi MT, Barac A, et al. The seroprevalence rate and population genetic structure of human cystic echinococcosis in the Middle East: A systematic review and meta-analysis. Int J Surg. 2018;51:39-48. doi: 10.1016/j.ijsu.2018.01.025. [PubMed: 29367032].

4. Alamer A, Aldhilan A, Makanjuola D, Alkushi A. Preoperative diagnosis of hydatid cyst of the breast: A case report. Pan Afr Med J. 2013;14:99. doi: 10.11604/pamj.2013.14.99.2396. [PubMed: 23717714]. [PubMed Central: PMC3664866].

5. Arikan S, Yucel AF, Barut G, Kocakusak A. Hydatid disease in the breast: A case report. Acta Chir Belg. 2004;104(4):473-5. [PubMed: 15469169].

6. Cancelo MJ, Martin M, Mendoza N. Preoperative diagnosis of a breast hydatid cyst using fine-needle aspiration cytology: A case report and review of the literature. J Med Case Rep. 2012;6:293. doi: 10.1186/17521947-6-293. [PubMed: 22973941]. [PubMed Central: PMC3485117].

7. Uncu H, Erekul S. Hydatid cyst of the breast. Acta Chir Belg. 2007;107(5):570-1. [PubMed: 18074924].

8. Sharafi SM, Rostami-Nejad M, Moazeni M, Yousefi M, Saneie B, Hosseini-Safa A, et al. Echinococcus granulosus genotypes in Iran. Gastroenterol Hepatol Bed Bench. 2014;7(2):82-8. [PubMed: 24834298]. [PubMed Central: PMC4017566].

9. Kamali NI, Raza MH, Rab AZ, Akhtar S. Isolated hydatid cyst of the breast: A case report. Indian J Surg. 2013;75(Suppl 1):150-1. doi: 10.1007/s12262-012-0475-y. [PubMed: 24426546]. [PubMed Central: PMC3693278].

10. Masroor I, Azeemuddin M, Khan S, Barakzai A. Hydatid disease of the breast. Singapore Med J. 2010;51(4):e72-5. [PubMed: 20505900].

11. Motie MR, Ghaemi M, Aliakbarian M, Saremi E. Study of the radical vs. Conservative surgical treatment of the hepatic hydatid cyst: A10-year experience. Indian J Surg. 2010;72(6):448-52. doi: 10.1007/s12262-0100163-8. [PubMed: 22131653]. [PubMed Central: PMC3077205].

12. Brunicardi FC, Andersen DK, Billiar TR, Hunter JG, Jeffrey. B . Schwartz's principles of surgery ABSITE and board review, 10/e. 2016.

13. Moro PL. Epidemiology and control of echinococcosis. In: Baron EL, editor. Uptodate2017. 2017.

14. Motie MR, Rezapanah A, Pezeshki Rad M, Razavian H, Azadmand A, Khajeh M. Primary localization of a hydatid cyst in the latissimus dorsi muscle: An unusual location. Surg Infect (Larchmt). 2011;12(5):401-3. doi: 10.1089/sur.2010.077. [PubMed: 22004438].

15. Sheikh S, Akhter R, Bhat S, Wani R. Primary hydatid disease of breast: A case report. J Parasit Dis. 2017;41(3):908-11. doi: 10.1007/s12639-0170903-9. [PubMed: 28848301]. [PubMed Central: PMC5555947].

16. Koc A, Sarici IS, Vurdem UE, Karabiyik O, Gumus UO. Unusual presentation of hydatid cyst in breast with magnetic resonance imaging findings. Case Rep Med. 2017;2017:6237435. doi: 10.1155/2017/6237435. [PubMed: 28167966]. [PubMed Central: PMC5266840 publication of this paper].

17. Gupta S, Verma DK, Prikshit RK. A rare presentation of hydatid disease: Breast lump. J Case Rep. 2014;4(2):331-3. doi: 10.17659/01.2014.0083.

18. Kumar A, Bhowmik A. Clinicoradiological study of Breast hydatid: Our experience. Imperial Interdiscip Res. 2017;3(5). 